\title{
Symposium III
}

Computerization and Image Analysis in Toxicologic Pathology 


\title{
THREE DIMENSIONAL ANALYSIS OF LIVER CELL FOCI
}

\author{
Katsumi Imaida, Masae Tatematsu*, Toshio Kato, and Nobuyuki Ito
}

First Department of Pathology, Nagoya City University Medical School, Mizuho-ku, Nagoya, 467 Japan and *Laboratory of Ultrastructure Research, Aichi Cancer Center Research Institute, Chikusaku, Nagoya 464, Japan

Computer graphics system can be used to investigate any histopathological lesions stereologically, as well as to evaluate the lesions quantitatively, such as numbers per unit area. Liver cell foci, especially glutathione S transferase $\mathrm{p}$-form (GST-P) positive foci, of rats have been used to assess modification of hepatocarcinogenesis, including promoting and inhibiting activities of test chemicals ${ }^{1,2}$. Since transformation data gained from 2-dimensional sections to 3-dimensional value (lesions per volume) have been recommended, the applicability of mathematical formulae for the calculation of number of foci per volume, and 3-dimensional demonstration of GST-P positive foci were examined.

F344 male rats were given a single i.p. injection of diethylnitrosamine at a dose of $200 \mathrm{mg} / \mathrm{kg}$ body weight, and 2 weeks later, they received test compounds, such as phenobarbital, $\mathbf{N}$-ethyl-N-hydroxyethylnitrosamine. All animals were subjected to partial hepatectomy at week 3, and sacrificed at week 8 . For 3-dimensional reconstruction of the liver, paraffin-embedded liver was serially sectioned ( $5 \mu \mathrm{m}$ thick) and immunohistologically stained with GST-P at 10 to $15 \mu \mathrm{m}$ steps (every 2 or 3 sections) for up to 150 sections. Several GST-P foci and nodules were selected and reconstructed 3-dimensionally with the aid of a computer graphics system (Spicca Computer System-TRI, Nippon Avionics Co., Ltd., Japan).

The results showed that both 2- and 3-dimensionally expressed quantitative results were found to adequately demonstrate the modifying potential of the chemicals on hepatocarcinogenesis. Furthermore, the stereological reconstruction revealed that the shape of GST-P positive foci, especially if relatively large, is not always spherical, but that many demonstrate irregular branching forms. Since the transformation formulae, such as Enzmann and Campbell's formulae, are based on the assumptions of spherical lesions, the assumptions are not always met $^{2,3}$.

These reconstruction technique with the aid of a computer graphics system can be used for investigation accurately any lesions such as liver cirrhosis and gastric tumors as well as liver cell foci.

\section{References}

1. Ito, N, Tsuda, H, Tatematsu, M, Inoue, T, Tagawa, Y, Aoki, T, Uwagawa, S, Kagawa, M, Ogiso, T, Masui, T, Imaida, K, Fukushima, $\mathrm{S}$, and Asamoto, $\mathrm{M}$ : Enhancing effect of various hepatocarcinogens on induction of preneoplastic glutathione S-transferase placental form positive foci in rat; an approach for a new medium-term bioassay system. Carcinogenesis $9: 387-389,1988$.

2. Ito, N, Imaida, K, Hasegawa, R, Tsuda, H. In: R.O. McClellan et al. (eds.), Rapid bioassay methods for carcinogens and modifiers of hepato-carcinogenesis. Crit Rev Toxicol CRC 19: 385-415, 1989.

3. Imaida, K, Tatematsu, M, Kato, T, Tsuda, H, and Ito, N : Advantage and limitations of stereological estimation of placental glutathione S-transferase-positive rat liver cell foci by computerized three-dimensional reconstruction. Jpn J Cancer Res 80 : 326-330, 1989. 
COMPUTER-AIDED VISUALIZATION OF 3-DIMENSIONAL TISSUE

MICROSTRUCTURES : CIRRHOSIS OF THE LIVER AND ITS MORPHOGENESIS

\author{
Tohru Takahashi, Yasuyuki Nakamura, and Ryoji Chiba \\ Department of Pathology, Research Institute for TB \& Cancer, Tohoku University, 4 Seiryomachi, \\ Aoba-ku, Sendai, 980 Japan
}

The 3-dimensional structure of an organ can be quite different from what it appears on a 2dimensional section. Thus, there are many aspects in pathology where one cannot obtain full understanding in the development of organ diseases if he fails to take a 3-dimensional viewpoint in the study of morphogenesis. Recently, such studies became greatly facilitated with the aid of graphics computers now available in various sectors of scientific research ${ }^{1}$. We show, in an analysis of cirrhosis developing from pre-cirrhotic diseases of the liver, how effectively one can rely on a computer system in unravelling the mechanisms involved. In addition, attempts will be shown at introducing computer-aid in the quantification of organ changes as another example of computerization in pathology.

A cirrhotic liver, whether that of man or of animal, has a specific skeleton of 3-dimensional network ${ }^{2}$. This reduces the problem of cirrhogenesis to how and by what mechanism this skeleton is formed at the initiation and develops during the course toward the final stage of disease. We made it clear in 3dimensional studies of human livers that the regenerating parenchymal nodules, while appearing on a 2 dimensional section as seemingly separate masses, are in reality connected with the adjacent ones in the form of nodular chains forming a 3-dimensional network with many loops. This skeleton proved to originate in a bridging hepatic necrosis which, creating a network of interstitium in the affected liver, molds the nodular network. What is essential is that the skeleton of scars emerges in such a way as to connect small portal and central veins contained in necrotic areas, bringing about the so-called $\mathrm{P}-\mathrm{C}$ bridgings. This explains why in cirrhosis of the common type, multiple connections between the portal and the hepatic venous trees are responsible for creating a network skeleton.

However, a problem remains. The bridging necrosis usually expresses a "zonal" distribution around either a central or a portal vein, which in itself is not susceptible of $\mathbf{P}-\mathbf{C}$ bridging. One may find "zonal" lesions in the livers of animals given cirrhogenic drugs where necrosis develops mainly centrilobularly, or in human livers with chronic hepatitis where the lesions are distributed around the portal areas. Thus the most basic question of cirrhogenesis comprises how liver necrosis, while presenting as "zonal" character, produces P-C bridgings. We show, as visualized in a computer display, processes of cirrhogenesis and skeleton formation, in the livers of man with chronic hepatitis and in those of rats given thioacetamide for up to nine months. This will demonstrate what a useful tool of pathology one can find in computerassisted 3-dimensional visualization.

\title{
References
}

1. Takahashi, T, Chiba, T, and Yaegashi, H: Three-D reconstruction of biostructures and its computerization : A possible extension of stereology (Keynote Lecture). Acta Stereol 6/III : 723-731, 1987.

2. Takahashi, T: Topological analysis of the morphogenesis of liver cirrhosis. Virchows Arch A 377: 189-201, 1978. 


\title{
DIGITIZED IMAGING DURING NECROPSY AND HISTOPATHOLOGY, A NEW TOOL IN COMPUTERIZED TOXICOLOGIC PATHOLOGY
}

\author{
Hannes Westen \\ Pathology Data Systems, Hauptstr. 77, 4132 Muttenz, Switzerland
}

Pathomorphologic examination is based on images. Recent technical development in real-time image digitizing has opened new fields for the pathologist involved in toxicologic pathology. Two main methods are available to capture images with subsequent transfer into the computer : scanning of conventional color slides or paper photographs and imaging by real-time video. For practical on-line operation, video is the method of choice. When commercially available 3-chip cameras are used, adequate image quality for diagnostic purposes is guaranteed. Technically, PAL, NTSC, or SECAM video signals are transformed into digitized information using special computer boards, such as a "Frame Grabber". The digitized image has a resolution of $640 \times 512$ pixels at 16.7 million colors. The file size is $480 \mathrm{~KB}$ and can be compressed to about $50 \mathrm{~KB}$ storage space and 256 colors for the routine computer hard disk, without losing critical information. When fully integrated into pathology software, image digitizing allows recording of necropsy lesions on a routine basis, with subsequent on-line display of necropsy images during tissue processing (trimming), histopathologic evaluation, reporting, and archiving. In histopathology, real-time image capturing allows fast recording of lesions both for the pathologist's own records and for official documentation. Compressed image files can be transferred via telephone over long distances within less than a minute. Furthermore, on-line display of images, when linked to glossary definitions, facilitate the pathologist's task to maintain consistent diagnostic standards during macro- and microscopic evaluation.

\section{SCIENTIFIC FINDINGS AND PUBLIC HEALTH IMPLICATIONS OF LONG- TERM CHEMICAL CARCINOGENESIS EXPERIMENTS}

James Huff

National Institute of Environmental Health Sciences Research Triangle Park, North Carolina, USA

Chemicals cause cancer. Fortunately not all chemicals are considered either potentially carcinogenic to humans ${ }^{1-3}$ or to animals ${ }^{4,5}$, and the proportion of chemicals eventually identified to cause cancer in experimental animals is forecast to be relatively low ${ }^{6}$. Those chemicals identified as being causally associated with cancers in humans have all been shown to cause cancer in laboratory animals; in every instance at least one site of cancer was common to both mammalian species ${ }^{7-9}$. This knowledge, together with patent similarities in mechanisms of carcinogenesis across species, led to the scientific logic that chemicals shown clearly to be carcinogenic in animals ${ }^{2,4,7-10}$ should be considered as being likely and anticipated to present cancer risks to humans ${ }^{1,3}$. So far 100-150 "agents" or exposures have been identified.

The carcinogenicity database used for this paper originated in the late 1960 s by the National Cancer Institute and since 1978 has been continued and made more comprehensive by the National Toxicology Program. The extensive files contain, among other sets of information, i) detailed pathology data from longterm (most often 24 month) carcinogenesis studies on more than 425 chemicals, ii) comprising nearly 
1,700 individual sex-species experiments, iii) with about 5,000 separate control and exposure groups, iv) containing collectively 250,000 laboratory rodents, $v$ ) having at least ten million tissue sections that have been evaluated for toxicity and carcinogenicity. Significantly, 200-300 pathologists have participated in this program. Using this data set we have 1) determined the concordance in carcinogenic responses between rats and mice to be $74 \%$, and between sexes to be $85 \%$ (rats) to $87 \%$ (mice) ; 2) discovered that using male rats and female mice would have identified correctly $95 \%$ of the positive or no evidence chemical carcinogenicity results obtained using the more extensive protocol; 3) established a historical control file of tumor incidence data ; 4) evaluated the false positive rate in the interpretation of carcinogenesis studies, concluding that this rate is probably no more than7-8\%; 5) compiled listings of chemicals having like carcinogenic target sites for each of the 35-40 organs or systems for which histopathology diagnoses have been recorded routinely ; 6) demonstrated that evaluation of site-specific carcinogenic effects are preferable to doing analyses based on overall (all sites combined) tumor rates ; 7) learned that few chemicals cause only benign tumors or only liver tumors, the most common target site for chemically induced cancers ; 8) identified key sources of variability in tumor rates in long-term carcinogenesis studies; 9) ascertained that corn oil gavage or gavage per se exhibits little if any adverse impact on long-term studies; 10 ) showed that a Salmonella multi-strain assay was as good as a battery of four short-term in vitro tests for predicting in vivo carcinogenicity, yet was only $66 \%$ concordant with an $89 \%$ positive predictivity and a $55 \%$ negative predictivity; 11) investigated the relationship between chemically induced toxicity and chemically associated carcinogenicity, finding that few chemicals cause tumors only at the highest exposure concentration and that toxicity does not typically correlate with carcinogenesis. These data and interpretations are most useful for maintaining a historic and objective perspective when evaluating the carcinogenicity of contemporary experiments, and for identifying potential carcinogenic hazards to humans.

Since 1971 the International Agency for Research on Cancer (IARC) has been evaluating the epidemiological and experimental evidence for carcinogenicity of chemicals, mixtures of chemicals, industrial processes, occupations, life-style and cultural habits, and exposure circumstances ("agents") ${ }^{1,2,7,8}$. Nearly 100 are now recognized as being or strongly implicated as being carcinogenic to humans. Of the less than 1,000 agents evaluated adequately for carcinogenicity in laboratory animals, a varying spectrum of data from studies on humans are available for only about $20-25 \%$. So far, 61 agents are linked unequivocally to cancer in humans, and another 41 are considered as strongly suspected of being carcinogenic to humans. Not all of these have been or can be evaluated in animals because some are industrial processes or "occupations", some are environmental and cultural risk factors, and some are mixtures of agents. For those that can be studied experimentally, the qualitative concordance between humans and animals approaches unity, and in every case there is at least one common organ-site of cancer in both species.

For those chemicals, mixtures of chemicals, or exposure circumstances to which humans are exposed, the hallmark public health issue centers on what level of exposure, if any, will present no or little carcinogenic risks to individuals or populations being exposed ${ }^{11,12}$ ?? Obviously if humans are not exposed to a chemical carcinogen, then the expectation that that chemical will be a carcinogenic hazard to humans must be recognized as not being possible. Yet, even this apparent comfort might be short-lived since other laboratory chemical curiosities or industrial intermediates have subsequently been given wide-spread human exposure : e.g., vinyl chloride ; methyl isocyanate ; 2, 3, 7, 8-TCDD ; 1, 3-butadiene. Soberingly however, occupationally associated cancers will continue to be discovered long into the future ${ }^{13}$.

\section{References}

1. IARC: IARC Monographs on the Evaluation of Carcinogenic Risks to Humans : Overall Evaluations of Carcinogenicity: An Updating of IARC Monographs: 1 to 42, Suppl 7. International Agency for Research on 
Cancer, Lyon, 440 p, 1987.

2. IARC: Monographs on the Evaluation of Carcinogenic Risks to Humans. Vols 1-54. IARC, Lyon, $1972-1992$.

3. NTP/DHHS. Sixth Annual Report on Carcinogens, National Toxicology Program, Department of Health and Human Services, Research Triangle Park, North Carolina, 746 pp. [copies available upon request], 1991.

4. Huff, JE and Haseman, JK : Long-term chemical carcinogenesis experiments for identifying potential human cancer hazards : Collective database of the National Cancer Institute and National Toxicology Program (1976-1991). EHP, 1991 in press.

5. Fung, V, Huff, JE, Weisburger, E, and Hoel, DG : Retrospective evaluation of the NCI/NTP nomination and selection processes of 382 chemicals studied for long-term carcinogenicity. AACR Abstract. 83rd Mtn. Am Assoc Cancer Research, San Diego, California (20-23 May 1992), 1992.

6. Huff, JE and Hoel, DG : Hazard identification: Perspective and overview on the concepts and value of the initial phase in the risk assessment process of cancer and human health. Scand J Work Environ Health (1992 in press)

7. Tomatis, L: The predictive value of rodent carcinogenicity tests in the evaluation of human risks. Ann Rev Pharmacol Toxicol 19: 511-530, 1979.

8. Tomatis, L, Aitio, A, Wilbourn, J, and Shuker, L : Human carcinogens so far identified. Jpn J Cancer Res 80 : $795-$ 807, 1989.

9. Huff, JE and Rall, DP : Relevance to humans of carcinogenesis results from laboratory animal toxicology studies, 433-440 and 453-457. In : Last, JM and Wallace, RB (eds), Maxcy-Rosenau-Last's Public Health and Preventive Medicine. 13th Ed. Appleton and Lange, Norwalk, CT, 1257 pp, 1992 in press.

10. Rall, DP, Hogan, MD, Huff, JE, Schwetz, BA, and Tennant, TW : Alternatives to using human experience in assessing health risks. Ann Rev Public Health 8 : 355-385, 1987.

11. NRC/NAS (National Research Council/National Academy of Sciences) : Risk Assessment in the Federal Government : Managing the Process. National Academy Press, Washington, pp. 189, 1983.

12. Hoel, DG, Taylor, JA, and Huff, JE: Data, assumptions, and controversies in qualitative cancer risk assessments. Environ Health Perspect 100. (1992 in press).

13. Doll, R: Occupational cancer : problems in interpreting human evidence. Ann Occup Hyg 28 291-305, 1984.

\section{NEW STRUCTURAL CONCEPTS FOR PREDICTING CARCINOGENICITY IN RODENTS : AN ARTIFICIAL INTELLIGENCE APPROACH}

\section{Gilles Klopman}

Department of Chemistry, Case Western Reserve University, Cleveland Ohio 44106, USA

Structure-Activity studies are important in many areas of mechanistic and exploratory chemistry. They are based on the premise that a relationship may exist between the chemical, or biological properties of a series of molecules and some of their independently observed physical or chemical properties. One of the problems with this kind of approach for the study of toxicity, particularly genotoxicity, is the inability of the systems to handle the diversity of the molecular structures that elicit activity. While in some cases, it is possible to identify congenericity, e.g. N-Nitroso-amines, Polycyclic Aromatic Hydrocarbons, etc..., more than often, the molecules have unique or cpmplex structures and their activity cannot easily be related to an identifiable structural functionality. In these cases, very little hope existed to find a satisfying solution, until the development of knowledge-based systems.

Knowledge-based systems are computer programs aimed at organizing relevant experimental data for the purpose of helping a user make a decision about real world problems. We describe one such method, CASE (Computer Automated Structure Evaluator) ${ }^{1-4}$, designed for the specific purpose of organizing toxicological data obtained from the evaluation of diverse chemicals. CASE automatically identifies molecular sub-structures that have a high probability of being relevant or responsible for the observed carcinogenicity of a learning set comprised of a mix of active and inactive molecules of diverse composition $^{5,6}$. CASE differs from other SAR techniques ${ }^{7}$ in that it is completely automatic and learns directly from the crude data. It selects its own descriptors from the practically infinite number of possible structural assemblies and creates an ad hoc dictionary without human intervention. This is an important 
feature as other SAR procedures are primarily interactive between the operator and the computer, wherein the operator selects possible descriptors that are part of a fixed panel. Unless the correct descriptors are included, and the appropriate one may very well not be part of the panel, then the correlations obtained are not good.

In order to create its knowledge, CASE must be trained with experimental data. For rodent carcinogenicity, the training set we used was the Rodent Carcinogenicity Data Base developed by the National Toxicology Program (NTP) of the US National Institute of Environmental Health Science . $^{8}$ This database contains approximately 250 chemicals tested by the NTP using a strict and consistent protocol. ${ }^{9}$ Once CASE has learned and organized the data, new, untested molecules can be submitted to the program, and an expert prediction of the potential carcinogenicity of the new molecule is obtained. The ability of the method to predict carcinogenic activity of unknown molecules will be discussed and compared to the results of equivalent experimental assays. It is found that these methods offer a viable alternative to both in vitro and animal bioassays.

\title{
References
}

1. Klopman, G : : Artificial-intelligence approach to structure activity study: Computer automated stucture evaluation of biological-activity of organic-molecules. J Am Chem Soc 106; 7315-7321, 1984

2. Klopman, G and Rosenkranz, HS : Structural requirements for the mutagenicity of environmental nitroarenes. Mutat Res 126; 227-238, 1984.

3. Klopman, $\mathbf{G}$ and Contreras : Use of artificial intelligence in structure-activity correlations of anticonvulsant drugs. Mol Pharmacol 27: 86, 1984.

4. Klopman, G: McKinney, J, ed., Health and Perspectives. Vol., 61., pp. 2691985.

5. Klopman, G, Namboodiri, K, and Kalos, A: The Molecular Basis of Cancer, Rein, R, Ed, A.R. Liss, Inc., 1985.

6. Rosenkranz, HS and Klopman, G : Genetic Toxicology of Environmental Chemicals, Part A : Basic Principles and Mechanisms of Action, 1986.

7. Frierson, M, Klopman, G, and Rosenkranz, HS : Structure-activity-relationships (SARS) among mutagens and carcinogens: A review. Environ Mutagen 8; 283-327, 1986.

8. Haseman, JK, Huff, JE, Zeiger, E, and McConnell, EE : Comparative results of 327 chemical carcinogenicity studies. Environ Health Perspect 74; 229-235, 1987.

9. Sontag, JM, Page, NP, and Saffiotti, U.: Guidelines for Carcinogenicity Bioassay in Small Rodents (National Cancer Institute Carcinogenesis Technical Report Series No. 1, DHEW Publication No. NIH 76-801). Washington, 1976.

\section{THE HANNOVER TUMOR REGISTRY DATABASE}

\author{
Ulrich Mohr ${ }^{1}$ and Gerd Morawietz ${ }^{2}$ \\ 'Hannover Medical School, Institute of Experimental Pathology, Konstanty-Gutschow-Str. 8, DW- \\ 3000 Hannover 61, Federal Republic of Germany \\ ${ }^{2}$ Fraunhofer Institute of Toxicology and Aerosol Research, Nikolai-Fuchs-Str. 1, DW-3000 Hannover \\ 61, Federal Republic of Germany
}

A computerized database of histopathological data obtained from control rats of various strains was established in a joint venture between 13 chemical and pharmaceutical companies from Germany and Switzerland. The purpose of this continuously expanding Hannover Tumor REGISTRY Data Base is to improve the evaluation and interpretation of the results of long-term studies with laboratory rodents. The system currently contains more than 10,000 diagnoses obtained from about 3,500 rats which were involved in 27 long-term studies. Besides the histopathological diagnoses of tumors and relevant preneoplastic lesions, the database includes detailed information on the maintenance and environmental conditions of each particular study (e.g. caging, diet, bedding, etc.) and on the status of each individual 
animal (e.g. strain, breeder, sex, age, body weight, etc.).

To facilitate a comparison of data collected in different studies and different laboratories and evaluated by different pathologists, a systematized nomenclature with standardized terms for lesions and organs and well-defined diagnostic criteria was developed together with the database ${ }^{1,2}$. All descriptions are given by keyword headings to be used as a directory. They summarize the essential diagnostic features and give criteria for the differentiation of hyperplastic lesions, benign and malignant tumors. The criteria have been validated in many slide sessions and panel meetings of the cooperating pathologists in order to make them acceptable for routine toxicological pathology.

To ensure that each individual diagnosis entered into the REGISTRY data base is consistent with the defined nomenclature, all findings are pre-checked by experienced pathologists at the Fraunhofer Institute in Hannover and selected cases (questionable diagnoses, borderline cases, interesting or rare lesions) are presented to a panel of pathologists of the participating companies. This procedure guarantees that future evaluations of the collected data will yield reliable and consistent results.

The complete REGISTRY nomenclature consists currently of about 600 pages of text. A special computer program named RENI (REGISTRY Nomenclature Information system) was developed for quick retrieval of specific information from this nomenclature. The program, which is designed to run on any standard Personal Computer without the need for additional expensive database software, contains the complete nomenclature lexicon and all diagnostic criteria. The REGISTRY Electronic Slide Library extension to RENI, which is a pathology video disk system, is currently under development. This technology will allow the storage of about 30,000 different high quality pictures on one video disk with an average access time for an individual slide in the order of one second. In this way, each single term of the REGISTRY nomenclature can be visually explained with several pictures of histopathological reference slides showing, for example, different magnifications and/or different staining methods.

First evaluations of the information stored in the database indicate a probable relationship between the prevalence of tumors and different husbandry parameters or genetic parameters of the strains used ${ }^{3}$. This demonstrates the need to have all kinds of additional information besides the histopathological diagnoses when using historical control data for the interpretation of animal studies.

\section{References}

1. Mohr, U, Bader, R, Ernst, H, Ettlin, R, Gembardt, C, Harleman, JH, Hartig, F, Jahn, W Kaliner, G, Karbe, E, Kaufmann, W, Krieg, K, Krinke, G, Küttler, K, Landes, C, Mettler, F Morawietz, G, Notman, J., Püschner, H, Qureshi, S, Reznik, G., Rittinghausen, S, Tuch, K, Urwyler, H, Weisse, G, Weisse, I, and Zehnder, J: Tumor REGISTRY Data Base : Suggestions for a systematized nomenclature for pre-neoplastic and neoplastic lesions in rats. Exp Pathol 38: 1-18, 1990.

2. Morawietz, G, Rittinghausen, S, and Mohr, U: Tumor REGISTRY Data Base : Advantages using a Systematized Nomenclature. Schweiz Arch. Tierheilk. 132: 448-449, 1990.

3. Morawietz, G and Rittinghausen, S: Variations in prevalence of endocrine tumors among different colonies of rats? A retrospective study in the Hannover Tumor REGISTRY Data Base. Arch Toxicol, in press. 